\title{
Cáncer gástrico: características epidemiológicas, clínicas y patológicas de los pacientes atendidos en el Hospital Universitario de Neiva entre enero del 2007 y diciembre del 2012
}

\section{Gastric cancer: epidemiological, clinical and pathological characteristics of patients treated in the university hospital of Neiva, between january 2007 and december 2012}

\section{Alejandro González' Ernesto Benavides², Dagoberto Santofimio ${ }^{3}$, Francisco Gil ${ }^{4}$}

\begin{abstract}
Resumen
El cáncer gástrico es la segunda causa de muerte por enfermedad maligna en el mundo siendo diagnosticado más frecuentemente en estadios avanzados con una incidencia y prevalencia elevada. Métodos: Se realizó un estudio observacional descriptivo que tiene como objetivo la descripción de variables epidemiológicas, patológicas y clínicas en pacientes con cáncer gástrico, en el Hospital Universitario de Neiva entre enero del 2007 y diciembre del 2012. Se estudiaron variables demográficas, clínicas, tipo histológico, diagnósticas y de tratamiento. Resultados: Se encontró 396 pacientes con adenocarcinoma gástrico, con mayor frecuencia de presentación en hombres con una relación hombre: mujer de 2:1 (promedio de edad de 59,7), el 85\% provenían del Departamento del Huila siendo Neiva el municipio más prevalente, el síntoma principal fue epigastralgia $(59,35 \%)$, seguida de pérdida de peso $(46,38 \%)$. El tipo histológico más frecuente en hombres fue intestinal $(49.6 \%)$ y en mujeres fue difuso (36.9). Se localizó con más frecuencia el adenocarcinoma gástrico en el tercio distal 222 pacientes $(56.6 \%)$. El método de apoyo diagnóstico más utilizado fue la biopsia gástrica $(89,4 \%)$, se detectó la enfermedad en estadios avanzados III $85(21,5 \%)$ y IV en $149(37,6 \%)$. Fallecieron 167 pacientes $(42,2 \%)$, de los cuales 97 a causa de enfermedad oncológica. Conclusiones: El cáncer gástrico es una patología con una alta incidencia y prevalencia en el Departamento del Huila y el Hospital Universitario de Neiva es un centro de referencia para los pacientes por lo cual debe continuar adelantando estrategias para mejorar la detecciones tempranas y realizar un estudio prospectivo que permita identificar y caracterizar los factores de riesgo para el desarrollo de cáncer gástrico.
\end{abstract}

Palabras clave: epidemiología, neoplasias gástricas, adenocarcinoma gástrico

\section{Abstract}

Gastric cancer is the second leading cause of death in the world from a malignant disease and is most commonly diagnosed at an advanced stage with high incidence and prevalence rates. Method: A descriptive, observational study was carried out with the aim of studying the epidemiological, clinical and pathological

1. MD. Rural en investigación, Universidad Surcolombiana, Hospital Universitario de Neiva. Colombia

2. MD. Especialista Hematología y Oncología, Hospital Universitario de Neiva. Colombia

3. MD. Ph.D(c), Epidemiología y Salud Pública. Docente Universidad Surcolombiana, Hospital Universitario de Neiva Colombia.

4. Estudiante de Medicina Universidad Surcolombiana, Neiva. Colombia. Correspondencia: Alejandro González. Correo electrónico: Alejo.g.motta@gmail.com Recibido: 30/12/2013-Revisado: 06/11/2014-Aceptado: 01/11/2015 
R.F.S Revista Facultad de Salud

Julio-Diciembre de 2015;7(2):23-28
Cáncer gástrico: características epidemiológicas, clinicas y patológicas de los pacientes atendidos en el Hospital Universitario de Neiva...

variables in patients with gastric cancer in the University Hospital of Neiva between January 2007 and December 2012. The study examined histological type and demographic, clinical, diagnostic and treatment variables. Results: The study found 396 patients with gastric adenocarcinoma, occurring more frequently in men, with a male to female ratio of $2: 1$. The average age was $59.7 .85 \%$ came from the department of Huila, Neiva being the most common municipality. The main symptom was epigastralgia (59.35\%), followed by weight loss (46.38\%). The most common histological type in men was intestinal (49.6\%) and diffuse in women $(36.9 \%)$. The gastric adenocarcinoma was more commonly found in the distal third, in 222 patients (56.6\%). The most widely used diagnostic method was the gastric biopsy (89.4\%). The disease was detected at advanced stage III in 85 patients (21.5\%) and at stage IV in 19 (37.6\%). 167 patients $(42.2 \%)$ died, the oncological disease was the cause of death in 97 . Conclusions: Gastric cancer is a pathology with high incidence and prevalence rates in the department of Huila. The University Hospital of Neiva is a point of reference for patients and therefore must continue developing strategies to improve early detection and carry out a prospective study in order to identify and characterise the risk factors of the development of gastric cancer.

Key words: epidemiology, gastric neoplasms, gastric adenocarcinoma

\section{Introducción}

El cáncer gástrico es una neoplasia que se origina en el estómago y abarca diferentes tipos histológicos entre los que se encuentra el adenocarcinoma, el linfoma no Hodgkin y los tumores carcinoides. Sin embargo el más frecuente con una amplia diferencia sobre los demás es el adenocarcinoma ${ }^{1-3}$ por esta razón cuando se habla de cáncer gástrico o carcinoma gástrico se hace referencia al adenocarcinoma gástrico.

En el 2012 se estimo una incidencia de 952.000 nuevos casos de cáncer gástrico en el mundo representando el quinto cáncer más común; aproximadamente el $70 \%$ de los casos se presentan en países en desarrollo ${ }^{4}$. En Colombia entre los años 2004-2006 y entre los años 2007-2011 el cáncer de estómago represento la segunda causa de incidencia en hombres y la cuarta en mujeres, ocupando la primera causa de muerte por cáncer en hombres y la tercera en mujeres ${ }^{5,6}$. Los Departamentos en Colombia con mayores tasas ajustadas de incidencia por cáncer de estómago en hombres en el periodo entre el 2007-2011 fueron, en orden decreciente: Tolima, Caldas, Boyacá y el Huila ${ }^{5}$ en el Departamento de Nariño en el municipio de La Cruz se ha reportado una de las incidencias mas altas a nivel mundial?

Diferentes factores de riesgo se han asociado con el desarrollo de cáncer gástrico entre ellos esta el consumo pesado de alcohol, un alto consumo de sal, consumo de carnes rojas, carnes preservadas, el uso de cigarrillo, la infeccion por Helicobacter Pylori y por el Virus del Epstein Barr ${ }^{4,8,9}$.

El cáncer gástrico presenta tambien un componente de susceptibilidad genética que se expresa en los individuos con grupo sanguineo tipo A que tienen un $20 \%$ mas de riesgo para desarrollar cáncer comparado con las personas que poseen otros grupos sanguíneos ${ }^{4}$, también se presenta en las personas con mutaciones de la proteína de adhesión celular E-cadherina en las que se ha encontrado una predisposición para el desarrollo de cáncer gástrico de tipo difuso con presentación familiar ${ }^{10}$ también en los polimorfismos de los genes de la interleucina $1 \mathrm{~B}$ y su receptor antagonista
IL 1RN se han asociado con el desarrollo de cáncer gástrico en caucásicos ${ }^{11}$

Este artículo describe las características sociodemográficas, diagnósticas, clínicas y patológicas de los pacientes con cáncer gástrico en la ciudad de Neiva, Colombia, en el periodo comprendido entre enero del 2007 y diciembre del 2012.

\section{Materiales y métodos}

Se diseñó un estudio observacional descriptivo, que se llevó a cabo en el Hospital Universitario de Neiva, Colombia. Se identificaron pacientes que fueron diagnosticados con cáncer gástrico entre enero de 2007 y diciembre de 2012. La información fue obtenida de los registros de la unidad de cancerología del hospital y de los reportes de patología de la institución en el periodo de investigación. El estudio obtuvo aprobación del comité de ética del Hospital Universitario de Neiva

La información epidemiológica, patológica y clínica de los pacientes se obtuvo de la historia clínica física. Los pacientes incluidos tenían confirmación histológica por biopsia gástrica o espécimen de patología extrainstitucional o intra-institucional con diagnostico de cáncer gástrico infiltrante o "in situ". Los variables incluidas en el estudio fueron: sexo, tipo de seguridad social, edad de diagnóstico, lugar de vivienda actual, tipo de cáncer, síntomas y signos de la enfermedad, método diagnóstico histológico utilizado, clasificación de Borrmann, método utilizado para establecer el tipo histológico, compromiso nodular regional, el tipo histológico final fue tomado de los resultados del espécimen de quirúrgico, cuando este no estaba disponible, se utilizó el resultado de la biopsia o la citología del líquido peritoneal, grado histológico, elementos de apoyo diagnóstico, localización del tumor, clasificación TNM, estadio de la enfermedad, presencia y localización de metástasis, tratamientos neoadyuvantes, primarios y adyuvantes utilizados, uso de laparoscopia para estadificación y estado vital del paciente durante el periodo del estudio. Toda la información fue reco- 
lectada por un médico general y un estudiante de pregrado de medicina.

Las variables fueron registradas en un archivo de Microsoft Excel ${ }^{\circledR}$ previamente codificado. La información fue resumida de manera descriptiva para las variables cualitativas y cuantitativas. Para estas últimas, se evaluó el supuesto de normalidad utilizando gráficas y la prueba de Shapiro-Wilk. Toda la información fue analizada en el programa estadístico Epi-Info 7 (versión 7.1.4.0) (CDC, EE.UU.).

\section{Resultados}

Se detectaron 396 pacientes con diagnóstico histopatológico de cáncer gástrico en el periodo a estudio. El 67,2\% de la población fue masculina. El promedio de edad de diagnóstico 59,7 años (Desviación estándar DE 14,3 años). La procedencia más común fue el Departamento del Huila $(85,6 \%)$ y en menor proporción el Departamento de Caquetá $(11,6 \%)$. Otros sitios fueron Cauca $(0,7 \%)$, Putumayo $(0,7 \%)$, Bogotá $(0,2 \%)$ y Tolima $(0,2 \%)$.

La frecuencia de los síntomas más comunes de la enfermedad fueron epigastralgia $(59,8 \%)$, pérdida de peso $(46,7 \%)$, vómito $(25,3 \%)$, dolor abdominal $(18,4 \%)$, adinamia $(16,7 \%)$, melenas $(13,9 \%)$, pirosis $(12,9 \%)$ y disfagia $(11,4 \%)$. En resumen se presentan las características clínicas e histopatológicas de los pacientes (Tabla 1, 2). El tipo histológico en hombres fue intestinal y en mujeres fue difuso (Tabla 3 ).

Tabla 1. Características clínicas de pacientes con diagnóstico de cáncer gástrico atendidos en el Hospital Universitario Hernando Moncaleano Perdomo (Neiva, Colombia) en el periodo 2007 a 2012 (n=396).

\begin{tabular}{|c|c|c|}
\hline Característica & $\mathrm{n}$ & $\begin{array}{l}\text { Frecuencia } \\
\text { (porcentaje) }\end{array}$ \\
\hline \multicolumn{3}{|l|}{ Método diagnóstico } \\
\hline Biopsia gástrica & 354 & 89,4 \\
\hline Espécimen quirúrgico & 177 & 44,7 \\
\hline Líquido peritoneal & 10 & 2,5 \\
\hline \multicolumn{3}{|c|}{ Principal localización del tumor } \\
\hline Tercio proximal & 75 & 18,9 \\
\hline Cuerpo & 69 & 17,4 \\
\hline Tercio distal & 222 & 56,6 \\
\hline Sin información & 30 & 7,5 \\
\hline \multicolumn{3}{|l|}{ Estadio } \\
\hline 1 & 23 & 5,8 \\
\hline$\|$ & 55 & 13,9 \\
\hline II & 85 & 21,5 \\
\hline IV & 149 & 37,6 \\
\hline Sin información & 84 & 21,2 \\
\hline
\end{tabular}

Tabla 2. Características histopatológicas de pacientes con diagnóstico de cáncer gástrico atendidos en el Hospital Universitario Hernando Moncaleano Perdomo (Neiva, Colombia) en el periodo 2007 a 2012 ( $n=396)$.

\begin{tabular}{lcc}
\hline Característica & $\mathbf{n}$ & $\begin{array}{c}\text { Frecuencia } \\
\text { (porcentaje) }\end{array}$ \\
\hline Clasificación de Borrmann & & \\
Tipo I & 9 & 2,3 \\
Tipo II & 46 & 11,6 \\
Tipo III & 133 & 33,6 \\
Tipo IV & 32 & 8,1 \\
Tipo V & 38 & 9,6 \\
Sin información & 138 & 34,9 \\
\hline \hline
\end{tabular}

\section{Tipo histológico}

Difuso

$\begin{array}{cc}180 & 45,4 \\ 123 & 31,0 \\ 45 & 11,3 \\ 28 & 7,1 \\ 8 & 2,0 \\ 2 & 0,1 \\ 10 & 2,5\end{array}$

Indiferenciado

Sin otra especificación

Mucinoso

In situ

0,1

Otros

Grado histológico

$\begin{array}{lcc}\text { Grado } 1 & 61 & 15,4 \\ \text { Grado } 2 & 102 & 25,8 \\ \text { Grado } 3 & 176 & 44,4 \\ \text { Grado 4 } & 2 & 0,5 \\ \text { Sin información } & 55 & 13,9\end{array}$

Tabla 3. Distribución del tipo histopatológico según sexo en pacientes con diagnóstico de cáncer gástrico atendidos en el Hospital Universitario Hernando Moncaleano Perdomo (Neiva, Colombia) en el periodo 2007 a 2012 ( $n=396)$.

\begin{tabular}{lcccc}
\hline Tipo histológico & \multicolumn{2}{c}{ Hombre } & \multicolumn{2}{c}{ Mujer } \\
\hline & $\mathrm{n}$ & $\%$ & $\mathrm{n}$ & $\%$ \\
\hline Intestinal & 132 & 49,6 & 48 & 36,9 \\
Difuso & 69 & 25,9 & 54 & 41,5 \\
Indiferenciado & 26 & 9,8 & 19 & 14,6 \\
Sin otra especificación & 23 & 8,7 & 5 & 3,9 \\
Mucinoso & 6 & 2,3 & 2 & 1,5 \\
In situ & 2 & 0,8 & - & - \\
Otros & 8 & 3,0 & 2 & 1,5 \\
\hline
\end{tabular}


R.F.S Revista Facultad de Salud

Julio-Diciembre de 2015;7(2):23-28
Cáncer gástrico: características epidemiológicas, clinicas y patológicas de los pacientes atendidos en el Hospital Universitario de Neiva...
El $59,9 \%$ de los pacientes fueron tratados mediante procedimiento quirúrgico, de los cuáles 25 (10,5\%) fueron tratados con cirugía laparoscópica, $142(59,9 \%)$ con cirugía abierta y en $70(29,5 \%)$ casos no se conoció el tipo de abordaje. Se presentaron complicaciones quirúrgicas intraoperatorias en el 3,5\% de los pacientes operados y $9,5 \%$ presentaron complicaciones postoperatorias intrahospitalarias. El tratamiento adyuvante fue suministrado en $37,4 \%$ del total de pacientes, de los cuáles 13 pacientes recibieron quimioterapia exclusiva, 5 radioterapia exclusiva y 130 quimioterapia concomitante a radioterapia. E1 97,8\% de los pacientes tratados con radioterapia recibieron un esquema de $4500 \mathrm{cGy}$ en fracciones de 180 cGy. De los pacientes que recibieron quimioterapia como terapia adyuvante, el 77,5\% fue tratado con 5-fluoracilo más folinato de calcio.

Se presentó metástasis en 17 pacientes posterior al tratamiento. Un total de 167 pacientes (42,2\%) fallecieron, de los cuales 97 se debieron a la enfermedad oncológica. No hubo información disponible en $34,6 \%$ del total de pacientes.

\section{Discusión}

El cáncer gástrico es la primera causa de muerte por cáncer en hombres y la segunda en mujeres en Colombia ${ }^{5,6}$, El formato utilizado para la recolección de datos demográficos, clínicos, de diagnostico y tratamiento al compararlo con formatos validados para la recolección de registros hospitalarios de cáncer ${ }^{12}$ se encontró que en el formato utilizado recoge algunos de los datos e ítems diseñados en la metodología de dichos protocolos para recolección de datos especializados en pacientes con cáncer sin embargo el objetivo de este estudio esta mas enfocado en describir las características epidemiológicas, clínicas y patológicas de la población de pacientes con cáncer gástrico atendidos en el hospital Universitario de Neiva, que crear un registro de cáncer con las normas internacionales diseñadas para este fin, sin embargo se considera que este estudio puede ser un aporte importante para correlacionar datos de estudios donde si se utilizo una metodología validada para el registro de pacientes con cáncer en la unidad de cancerología del Huila desarrollado por Pardo et al. ${ }^{13}$ en el cual se encontraron 253 casos de cáncer de estomago entre el 2006-2008 de los cuales el $68,4 \%$ era hombres, la media de edad fue 59,5 años, el $57,7 \%$ pertenecía al régimen subsidiado, el 26,9\% al régimen contributivo y el $37,5 \%$ de los pacientes se encontraba en estadio IV lo cual se correlaciona con las datos encontrados en este estudio.

En el estudio realizado por el Instituto Nacional de Cancerología "Incidencia estimada y mortalidad por cáncer en Colombia, 2007-2011"6 se encontró el cáncer gástrico era la segunda neoplasia más frecuente en hombres después del cáncer de próstata y la cuarta neoplasia en mujeres. En la literatura mundial se encuentra que el $90 \%$ de todos 1 os tumores del estomago son malignos y el $95 \%$ de los tumores malignos son adenocarcinoma gástricos ${ }^{14}$ por esta razón el estudio busco caracterizar a los pacientes con diagnóstico de adenocarcinoma gástrico. En Colombia se estimo que entre el 2007-2011 se presentaron 3613 casos/ año de cáncer gástrico en hombres y 2342 casos/año de cáncer gástrico en mujeres así mismo se observo una mortalidad de 2767 hombres/año y 1770 mujeres/año a causa del cáncer gástrico en este periodo de tiempo ${ }^{6}$. Entre enero del 2007 y diciembre del 2012 en nuestro estudio se encontraron 396 casos de pacientes con cáncer gástrico con un promedio de 66 casos/año la incidencia estimada de cáncer gástrico en el Departamento del Huila es de 203 casos/año ${ }^{6,15}$ de lo que el hospital Universitario de Neiva atendería aproximadamente el $32,5 \%$ de los casos de cáncer gástrico en el departamento del Huila.

La clasificación y el tipo histológico reportado en las biopsias gástricas y patologías de especimenes quirúrgicos y biopsias de ganglios fue de difícil clasificación en el momento de la recolección de los datos debido a que existe muchas diferencias en la clasificación histológica del cáncer gástrico utilizado entre los patólogos y unidades de patología de la región por ello en algunos casos utilizan la clasificación de Lauren en otros la clasificación de la OMS o la de Nakamura, además los reportes de diagnóstico de adenocarcinoma gástrico indiferenciado en los cuales no se realizó inmunohistoquímica son de difícil clasificación para el análisis de datos, en otros casos el reporte de patología solamente refería "Adenocarcinoma gástrico" sin dar otro tipo de información complementaria, se considera que se pueden hacer esfuerzos para unificar los diagnósticos patológicos de la región que permitan y faciliten el desarrollo de estudios en cáncer gástrico en un futuro.

Al comparar los datos obtenidos con un estudio realizado en la ciudad de Bucaramanga por Bermúdez et al. ${ }^{16}$ se encontró que la localización mas frecuente del tumor fue antro-pilórica y la totalidad de los casos se diagnosticaron en estadio III y IV en nuestra población por el contrario la localización mas frecuente del tumor fue el antro gástrico seguido por el cuerpo y en estadio III y IV se encontró al 59,1\% de la población, llama la atención para nosotros que al parecer la detección de cáncer gástrico en hospital Universitario de Neiva se realizó en estadios tempranos en un mayor numero en comparación con la reportada en el hospital Universitario de Santander.

La mayoría de los pacientes en nuestro estudio tuvo diagnostico histológico de subtipo intestinal lo cual es importante ya que este subtipo histológico tiene mejor pronostico que el difuso ${ }^{17}$ y se asocia con menor frecuencia de resecciones quirúrgicas R1 (residuo histológico de tumor) ${ }^{18}$.

Los principales síntomas referidos por los pacientes en el momento del diagnóstico fueron epigastralgia, perdida de peso y vomito. El principal método diagnóstico utilizado fue la endoscopia de vías digestivas altas, la localización del tumor mas frecuente del tumor fue el antro gástrico seguido por el cuerpo del estómago.

En el momento del diagnóstico se encontró metástasis en el $37,6 \%$ de los pacientes y en estadios III y IV al $59,1 \%$ de los pacientes lo que evidencia el alto porcentaje de pacientes detectados en estadios avanzados, en Estados Unidos el $65 \%$ de los pacientes son detectados en 
estadios avanzados ${ }^{3}$, nuestros datos se correlacionan con esta estadística. En los países occidentales se requiere mejorar las técnicas de tamizaje y detección temprana ya que los estadios avanzados se correlacionan con una alta mortalidad y con una mediana de supervivencia de 10-12 meses $^{19}$ motivo por el cual representa la tercera causa de muerte por cáncer en el mundo en ambos sexos ${ }^{4}$; en nuestro estudio se encontró que $42,2 \%$ de los pacientes habían fallecido sin embargo no hubo información disponible en un grupo considerable de pacientes y al tratarse de un estudio retrospectivo descriptivo no tenia como objeto el cálculo de supervivencia.

\section{Limitaciones}

El estudio tiene limitaciones inherentes al diseño del mismo al ser un estudio observacional y descriptivo no determina factores de riesgo para el desarrollo de cáncer gástrico ni busco calcular supervivencia global ni cáncer especifica.

\section{Conclusiones}

El cáncer gástrico es una patología con una alta incidencia y prevalencia en el Departamento del Huila siendo el hospital Universitario de Neiva un centro de referencia en el sur del país. Se determinó por medio de un estudio observacional descriptivo que los pacientes con adenocarcinoma gástrico que consultaron son en su mayoría de sexo masculino con una relación hombre: mujer de $2: 1$, con una edad media de 59,7 años en el momento del diagnóstico, siendo más frecuente de tipo histológico intestinal en hombres y difuso en mujeres, donde el Huila es el sitio de procedencia de mayor proporción de la población estudiada. Los síntomas más frecuentes fueron epigastralgia y pérdida de peso. Se recomienda continuar adelantando estrategias para mejorar las detecciones tempranas y realizar un estudio prospectivo que permita identificar y caracterizar los factores de riesgo para el desarrollo de cáncer gástrico.

\section{Agradecimientos}

Los autores agradecemos al Hospital Universitario Hernando Moncaleano Perdomo de Neiva por la financiación económica en su totalidad y al Doctor Jesús Antonio Castro por la disposición recibida para llevar a cabo la realización de este estudio. Así mismo agradecemos al departamento de $\mathrm{Pa}-$ tología y la unidad de Cancerología por facilitar las herramientas que permitieron la recolección de los pacientes para el desarrollo de este proyecto.

\section{Referencias}

1. Japanese Gastric Cancer Association. Japanese classification of gastric carcinoma: 3rd English edition Gastric Cancer. 2011 Jun; 14(2):101-12.

2. Rotterdam H. Carcinoma of the stomach. In: Rotterdam $H$, Enterline HT. Pathology of the stomach and duodenum. New York: Springer-Verlag, 1989:1 42-204.
3. Dicken BJ, Bigam DL, Cass C, Mackey JR, Joy AA, Hamilton SM. Gastric adenocarcinoma: review and considerations for future directions. Ann Surg $2005 ; 241: 27-39$.

4. World Health Organization. GLOBOCAN 2012: Estimated Cancer Incidence, Mortality and Prevalence Worldwide [Internet]. Lyon, France: International Agency for Research on Cancer; 2012 [cited 2015 Oct 8]. Available from: http://globocan.iarc.fr/Pages/fact sheets_population.aspx

5. Pardo C, Cendales R. Incidencia estimada y mortalidad por cáncer en Colombia, 2002-2006. Bogotá; INC: 2010.

6. Pardo C, Cendales R. Incidencia mortalidad y prevalencia de cáncer en Colombia, 2007-2011. Bogotá; INC:2015.

7. Murillo R, Piñeros M, Suárez G, Garríos A, Pozo H. Atlas de mortalidad por cáncer de Colombia. Santa Fe de Bogotá: Instituto Nacional de Cancerología; 2003.

8. Bonequi P, Meneses-González F, Correa P, Rabkin CS, Camargo MC. Risk factors for gastric cancer in Latin America: a meta-analysis. Cancer Causes Control. 2013 Feb;24(2):217-31.

9. Tokunaga $M$, Land $C E$, Uemura $Y$, ef al. Epstein-Barr virus in gastric carcinoma. Am J Pathol. 1993;143:12501254.

10. Guilford P, Hopkins J, Harraway J, McLeod M, McLeod N, Harawira P, Taite H, Scoular R, Miller A, Reeve AE. Ecadherin germline mutations in familial gastric cancer. Nature. 1998 Mar 26;392(6674):402-5

11. Camargo MC, Mera R, Correa P, Peek RM Jr, Fontham ET, Goodman KJ, Piazuelo MB, Sicinschi L, Zabaleta J, Schneider BG. Interleukin-1 beta and interleukin-1 receptor antagonist gene polymorphisms and gastric cancer: a meta-analysis. Cancer Epidemiol Biomarkers Prev. 2006 Sep; 15(9):1674-87.

12. Jensen $O M$, Parkin DM, MacLennan $R$, Muir CS, Skeet RG. El registro hospitalario de cáncer. En: Registros de cáncer: principios y métodos.

13. Pardo C, Guzmán J, Rodríguez $O$. Cáncer en la Unidad de Cancerología de Huila, 2006-2008 Rev Colomb Cancerol. 2013;17(2):62-68

14. Schwartz G. Invasion and metastasis in gastric cancer: in vitro and in vivo models with clinical considerations. Semin Oncol. 1996;23:316-324.

15. Hernán Espejo Romero $\mathrm{H}$, Navarrete J. Clasificación de los Adenocarcinomas de estómago Rev Gastroenterol Perú 2003;23:199-212.

16. Bermúdez Carlos, Insuasty Jesús, Gamarra Germán. Grupo sanguíneo A y riesgo de cáncer gástrico en el Hospital Universitario de Santander (Bucaramanga, Colombia). Acta Med Colomb. 2006 Dec; 31 (4):400-410.

17. Qiu MZ, Cai MY, Zhang DS, Wang ZQ, Wang DS, Li $\mathrm{YH}, \mathrm{Xu} \mathrm{RH}$. Clinicopathological characteristics and prognostic analysis of Lauren classification in gastric adenocarcinoma in China. J Transl Med. 2013 Mar 6;11:58. doi: 10.1186/1479-5876-11-58. 
R.F.S Revista Facultad de Salud

Julio-Diciembre de 2015;7(2):23-28
Cáncer gástrico: características epidemiológicas, clínicas y patológicas de los pacientes atendidos en el Hospital Universitario de Neiva...
18. Stiekema J, Cats A, Kuijpers A, van Coevorden F, Boot $H$, Jansen EP, Verheij $M$, Balague Ponz $O$, Hauptmann $M$, van Sandick JW. Surgical treatment results of intestinal and diffuse type gastric cancer. Implications for a differentiated therapeutic approach?Eur J Surg Oncol. $2013 \mathrm{Jul} ; 39(7): 686-93$. doi: 10.1016/i.ejso.2013. 02.026. Epub 2013 Mar 15.
19. Digklia A, Wagner AD. Advanced gastric cancer: Current treatment landscape and future perspectives. World J Gastroenterol. 2016 Feb 28;22(8):2403-14. 